\title{
Simplifying Telerobotics: Wearability and Teleimpedance Improves Human-Robot Interactions in Teleoperation
}

\author{
Simone Fani ${ }^{\dagger, \ddagger, * *}$, Simone Ciotti ${ }^{\dagger, \ddagger, * *}$, Manuel G. Catalano ${ }^{\ddagger}$, Giorgio Grioli ${ }^{\ddagger}$, \\ Alessandro Tognetti ${ }^{\dagger, \S}$, Gaetano Valenza ${ }^{\dagger, \S}$, Arash Ajoudani ${ }^{\ddagger, \Lambda}$ and Matteo Bianchi ${ }^{\dagger, \S, \Lambda}$
}

\begin{abstract}
In recent years, wearability has become a new fundamental requirement for an effective and light-weight design of the human-robot interfaces. Among the different application fields, robotic tele-operation represents the ideal scenario that can largely benefit from the wearable paradigm, in order to reduce constraints to human workspace (acting as a master) and to enable an intuitive and simplified information exchange within the tele-operator system. This effective simplification is particularly important if we consider the interaction with synergy-inspired robotic devices, i.e. endowed with a reduced number of control inputs and sensors, with the goal of maintaining a simple control and communication between humans and robots. In this work, we present an integrated approach for augmented tele-operation where wearable hand/arm pose under-sensing and haptic feedback devices are combined with teleimpedance techniques, for a simplified yet effective position and stiffness control of a synergyinspired robotic manipulator in real-time. The slave robot consists of a Kuka lightweight robotic arm equipped with the Pisa/IIT SoftHand, both controlled in impedance to perform a drilling task, an illustrative example of dynamic tasks with environmental constraints. Experimental results on ten healthy subjects suggest that the proposed integrated interface enables the master to appropriately regulate the stiffness and pose of the robotic hand-arm system through the perception of interaction forces and vision, contributing to successful and intuitive executions of the remote task. The achieved performance is presented in comparison to the reduced versions of the integrated system, in which either teleimpedance control or wearable feedback are excluded.
\end{abstract}

\section{INTRODUCTION AND Motivation}

Bilateral robotic teleoperation or telerobotics, i.e., robotics at distance with force feedback to the user, represents a wellstudied problem in literature. The ideal goal is to enable the user not only to interact with the remote environment but also to perceive it as if touched directly. At the same time, due to the closed-loop operation of the system, the stability of the master-slave control loop should be also ensured. In literature, the trade-off between transparency and

This work is supported in part by the European Research Council under the Advanced Grant "SoftHands: A Theory of, Soft Synergies for a New Generation of Artificial Hands" (no. ERC-291166), by the EU FP7 project (no. 601165) "WEARable HAPtics for Humans and Robots (WEARHAP)", and by the EU H2020 projects (no. 645599)"SOMA: SOft MAnipulation" and "SoftPro: Synergy-based Open-source Foundations and Technologies for Prosthetics and RehabilitatiOn"(no. 688857).

${ }^{\dagger}$ Research Center "Enrico Piaggio", University of Pisa, Largo Lucio Lazzarino 1, 56126 Pisa, Italy

${ }^{\ddagger}$ Department of Advanced Robotics, Istituto Italiano di Tecnologia, via Morego, 30, 16163 Genova, Italy

$\S$ Department of Information Engineering, University of Pisa, via G. Caruso, 16, 56122 Pisa, Italy

** These authors contributed equally to this paper.

$\Lambda$ This work was equally supervised by these authors.

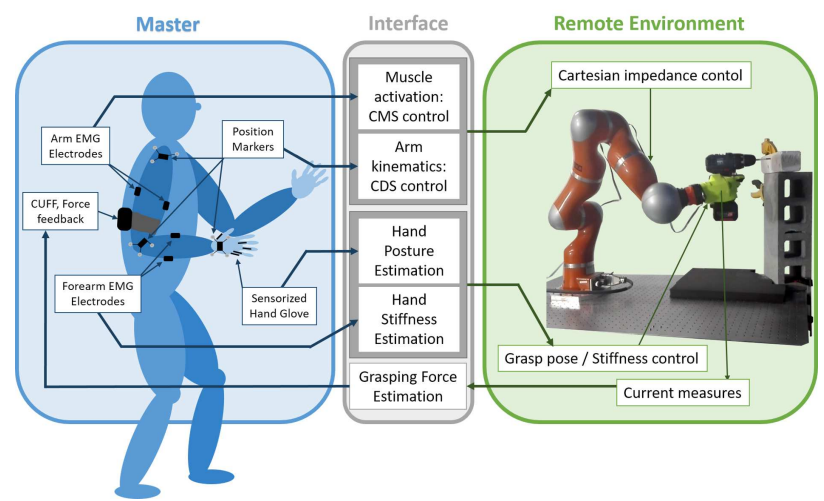

Figure 1: The integrated system and experimental set-up.

stability has been elucidated by several important studies [1], [2]. Indeed, while force feedback is essential to increase realism and effectiveness of the interaction in teleoperation tasks, it is challenging to avoid instabilities due for example to the presence of latencies in the communication channel. Among the different approaches for stable teleoperation, it is worth to mention passivity-based approaches (see e.g. [3]), which analyze the flow of energy of the system and imply a sufficient but not necessary and overly conservative condition for stability, and the Virtual Environment-based approach (see e.g. [4]), which generates a geometric and dynamic model of the remote physical environment. Other techniques rely on the substitution of grounded kinaesthetic force feedback [5] with other forms of feedback, such as visual/auditory or purely tactile [6], or to scale down the kinaesthetic feedback to satisfy passivity, at the cost of reducing transparency [7]. Visual-haptic feedback can be also combined with a virtualbased approach as in [8]. In our recent work, we proposed an alternative approach to ensure the stability in teleoperation, named teleimpedance, which consists in measuring and replicating the master's limb endpoint impedance on the slave robot in real-time [9]. Interestingly, such a direct mapping enables to exploit very useful characteristics of the human muscular-skeletal system, e.g., energy efficiency, resilience, and safety, which are typical of human behavior and that are specifically targeted by the development of soft robots [10]. Nevertheless, effective regulation of the interaction parameters in teleimpedance control requires a priori knowledge about the task or a good perception of the environment by the master. Although the integration of grounded force feedback into teleimpedance control would be a feasible option, this kind of feedback commonly imposes additional constraints to 
the human limb and severely limits its overall workspace.

To overcome such limitations and hence enhance transparency and intuitiveness of human robot interaction (HRI), wearable systems for haptic rendering and sensing gained an increasing attention in recent years [11]. Wearable haptic systems (WHS) are mainly thought to deliver tactile cues rather than kinaesthetic information, thus ensuring a good trade off between stability and transparency. These systems can indeed be comfortably worn by humans, carried around and integrated into their everyday life, with ideal applications related (but not limited) to assistive technologies, virtual reality, and telemanipulation of remote robotic systems [12].

Teleimpedance and wearable haptics/sensing can play a crucial role especially considering the overall simplification approach introduced by the concept of synergies, with special focus on hands. Synergies can be regarded as main covariation schemes in human hand joints defining principal patterns of actuation and sensing of human hands that reduce the burden for the control of human sensory-motor apparatus, see e.g. [13]. The synergy idea has hence found a fertile application field in robotics, inspiring the design, control and sensing of artificial systems with a reduced number of actuators, control inputs and sensors (See e.g. [14] for a review on this topic).

Towards the establishment of a teleoperation system that subsumes the advantages of wearable devices and remote impedance control, targeting under-actuated synergy-inspired robotic systems, this work integrates our recent results in wearable hand pose under-sensing and haptic feedback for the teleimpedance control of a robotic hand-arm system, see Fig. 1. The robotic system consists of a lightweight KUKA arm, equipped with the Pisa/IIT SoftHand, an anthropomorphic robot hand endowed with 19 DOFs actuated with only one motor, which ensures that the free-hand movement is in accordance with the first human grasping synergy, i.e. the most common pattern of actuation observed in human grasping [13]. SoftHand is also robust yet adaptable and can deform with the external environment to multiply its grasping capabilities [15]. The force feedback on the grasping force exerted by the telecontrolled robotic hand is delivered through a wearable device on the operator's arm [16]. At the same time, to increase the naturalness of human-robot interaction, we track human limb kinematics and the stiffness profiles through light-weight sensory systems that does not reduce the natural workspace of the master's limb. Furthermore, to enable a more ecological hand pose reconstruction (HPR), i.e. to provide natural inputs for the remote control of the artificial hand, a wearable undersensed solution is here exploited. Indeed, to allow natural interaction, it is particularly important to avoid cumbersome solutions, and hence to limit the number of sensors. It is also important to observe that HPR is provided for all the joints used for the kinematic hand description, but, through a suitable projection technique, only the contribution along the first grasping synergy implemented on the Pisa/IIT SoftHand is considered to command the position of the artificial manipulator in a more reliable manner.

Both the robotic hand and arm are controlled in torque. In KUKA the torque control is computed through torque sensing and actuation [17]. In the Pisa/IIT SoftHand the torque control is computed through current sensing and control on a custom control board. The tracking of the human hand movements in the direction of first kinematic hand synergy is achieved through an optimally designed under-sensed glove [18] and mapped to the robotic hand in real-time. Synchronously, an unidimensional index associates the co-contraction of the human grasp, estimated from one antagonistic pair of forearm muscles [17], to the SoftHand stiffness parameter. An interaction toque observer estimates the forces between the SoftHand and the object, which is fed back to the master using an upper arm wearable mechano-tactile device [16]. The tracking of the human right arm kinematics and its translational Cartesian stiffness components is achieved by reduced-complexity models that exploit minimum sensory data [19], [17] and replicated by the robotic arm's Cartesian impedance controller in realtime (decoupled from, but synchronous to, the control of the Softhand). The proposed interface enables the master to use arm configurations and muscular activations to regulate the pose and stiffness profiles of the robotic hand/arm system to generate task-required forces relying on the visual information and force feedback.

Fig. 2 shows a block diagram of the control scheme employed, which is explained in more details in the paper.

The manuscript is organized as follows. We will first describe the main pillars we leveraged upon for our integrated system and proposed simplification approach, i.e., (i) the robotic hand and arm system used for the experiments, (ii) common mode and configuration stiffness principles for realtime tracking of human arm endpoint and implementation for the control of the robotic hand; (ii) wearable under-sensing hand pose reconstruction system to track user's hand pose at the master side; (iii) wearable haptic feedback to convey information on the grasping force of the robotic hand. We will then describe the integration of these key ingredients and report on the results of the validation phase, whose main elements are represented in Fig. 2, carried out through preliminary experiments with human participants.

Main conclusions that can be drawn is that the integration proposed here contributes to successful and intuitive execution of remote tasks. The achieved performance is presented in comparison with reduced versions of the integrated system, where either teleimpedance control or wearable feedback are excluded.

It is worth to underline that the inspiring idea of our work is a generalized simplification strategy informed by the neuroscientific concept of synergies. This idea is, indeed, used for the development and control of the SoftHand and for the optimal design of HPR. Furthermore, the concept of synergies, intended in a broad sense, has also driven the implementation of teleimpedance and force-feedback. In teleimpedance, synergies led to a simplification of sensing components and methods, while in the implementation of force feedback it yields the possibility of rendering the overall force exerted by the SoftHand along its motion pattern relying on an indirect estimation of the force based on the current absorbed by the hand motor without using any extrinsic sensor. 


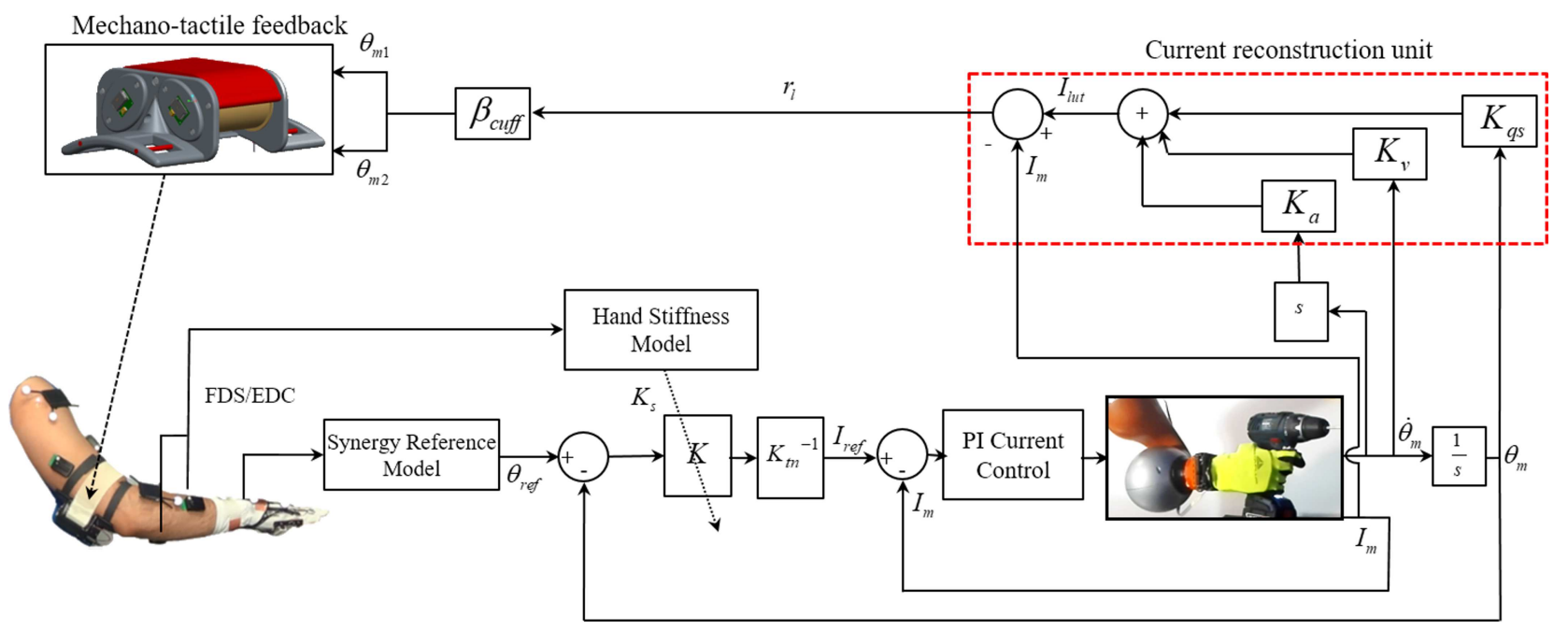

Figure 2: Block diagram of the integrated system. $\theta_{m}, \theta_{m 1}, \theta_{m 2}$ represent SoftHand motor and cuff motors angular positions, respectively. $\theta_{\text {ref }}$ is the synergistic reference configuration (or $\sigma$ ) commanded to the SoftHand through the glove-based kinematic reconstruction. $I_{m}$ is the current absorbed by the SoftHand motor and measured through current readings. $I_{l u t}$ represents the contribution to the reconstructed free-hand motion current due to the angular position, velocity and acceleration. $K_{q s}, K_{v}$ and $K_{a}$ represent the functions related to motor angular position, velocity and accelration, respectively. The term $r_{I}$ is used to compute the reference angular motor position of cuff motors through the proportional factor $\beta_{c u f f}$. For further details the reader is invited to refer to [16]. The other symbols are elucidated in the text.

\section{The Robotic Hand AND ARm System}

The Pisa/IIT SoftHand (SH) [15] is an anthropomorphic hand designed with 19 DOFs, 4 on each of the long fingers, and 3 on the thumb. The fingers are capable of flexion/extension as well as ab/adduction. For ab/adduction of the fingers and at the equivalent of the carpometacarpal joint of the thumb, traditional revolute joints were employed. The rest of the joints incorporate rolling contact joints with elastic ligaments, which ensure physiologically correct motions when actuated, but easily disengage on impact to allow safe interaction with humans while preserving the hand. The elastic ligaments also allow deformation while ensuring the hand returning to its original configuration. Afterwards this design enables the SH to softly interact with the environment and adapt to the items, exploiting the external constraints. A single tendon runs though all joints to simultaneously flex and adduct the fingers upon actuation. The hand is actuated by a single DC motor which moves the fingers on the path of the first synergy [13], hereinafter referred to as $S$ described in $\mathbb{R}^{19}$, allowing the SoftHand to mold around the desired object. For further details the reader is invited to refer to [15]. More information and CAD files of this robotic hand can be freely downloaded from Natural Machine Motion Initiative (NMMI) www.naturalmachinemotioninitiative.com/.

In this work, we used a myo-electric version of the Pisa/IIT SoftHand. More specifically, exploiting the concept of synergies that drive concurrent muscle activation, only one pair of antagonistic muscles (two surface EMG channels, from the major finger antagonist pair, i.e., the extensor digitorum communis (EDC) and flexor digitorum superficialis (FDS)) was used to drive the stiffness and postural synergy references tracked by the hand controller, as detailed later in the text, see also [17]. The hand is mounted over the wrist of a 7 DOF Kuka LWR IV+ arm. The position and end-point stiffness are controlled using the techniques detailed in the next section.

\section{COMMON-MOdE (CMS) AND CONFIGURATION DEPENDENT STIFFNESS (CDS) PRINCIPLES FOR TEAL-Time Tracking of the Human ARm Endpoint IMPEDANCE}

There are several important reasons why humans principally explore the control of arm configurations to perform tasks that require dynamic dexterity. One explanation for such a behaviour is the ergonomic efficiency of the postural adjustments in the generation of certain endpoint force manipulability or stiffness profiles in comparison to the muscular (co)activations [20]. Another important factor is the major contribution of the arm pose to effective modifications in the geometry of the endpoint stiffness ellipsoid. The latter can mathematically be described by transformations from muscle stiffness matrix $\left(K_{m}\right)$ to arm joint stiffness $\left(K_{J}\right)$, and consequently to the Cartesian stiffness matrix $\left(K_{c}\right)$ by ${ }^{1}$

$$
K_{c}(p, q)=J^{+T}(q)\left[J_{m}^{T}(q) K_{m} J_{m}(q)\right] J^{+}(q),
$$

with $K_{J}(p, q)=J_{m}^{T}(q) \hat{K}_{m}(p) J_{m}(q)$, and $p, q$ and $K_{m}$ being the muscle activation and joint angle vectors and muscle stiffness matrix, respectively. The quadratic effect of the arm kinematics through arm $J(q)$ and muscle $J_{m}(q)$ Jacobians on the Cartesian stiffness matrix is evident in this equation.

The tracking of the arm kinematics is achieved through passive marker motion capture system (Vicon) using the concept of arm tangle, as explained in [19]. Three rigid-body markers

\footnotetext{
${ }^{1}$ The effects of gravity and external load are neglected in this equation.
} 
can be attached to the hand, elbow and shoulder segments and used for the tracking of the arm Jacobian. Using the muscle attachment points [21], and the length variations over the joint angles, the muscle Jacobian can be computed online.

The effect of muscle stiffness matrix $\left(K_{m}\right)$ in joint and endpoint stiffness variations is commonly described using Hill's activation dynamic equations which provide a mapping between the muscular activities, usually measured by the EMGs, and the corresponding muscles' stiffness profiles. This, however, requires that the EMG activities of several muscles are processed and passed through a complex system of equations to account for the muscle stiffness matrix, eventually resulting in costly (need for several EMG sensors and amplification) and computationally intensive system. On the other hand, dense literature gives solid evidence on the existence of synergistic relationships between the arm monoand bi-articular muscle activities which realize a coordinated stiffening profile across the arm joints [22], [23]. As a result, co-contractions of the arm muscles mostly contribute to the modifications in the volume of the endpoint stiffness ellipsoid rather than to its direction [9]. This strategy is deemed to be exploited by the Central Nervous System (CNS) to solve for the motor complexity in an efficient and coordinated manner [24].

On these bases, in our recent work [19], the concepts of Common-Mode Stiffness (CMS) and ConfigurationDependent Stiffness (CDS) were proposed to associate the arm muscular activities and configurations to variations in the volume and major axes orientations of the arm endpoint stiffness ellipsoid, respectively. To design a real-time arm endpoint stiffness model, we presume that i) there exists a synergistic relationship between the arm muscle activities, and ii) each muscle activation contributes to the volume of the endpoint stiffness ellipsoid with a different ratio. Accordingly we propose $\hat{K}_{m}=a_{c c}(p) K_{s}$, with $K_{s}$ an experimentally identified time-invariant diagonal matrix which implements the contribution of each muscle to the active variations of the volume of the endpoint stiffness with a certain weight. The scalar and time-varying component, $a_{c c}(p)$, which is a function of the muscular activities, is multiplied to the muscle weights to compute the overall contribution of muscle activations to the volume of the endpoint stiffness ellipsoid. Obviously, depending on the choice of muscles that are used for the computation of active component $\left(a_{c c}\right)$, the identified scale matrix $K_{s}$ would differ. In this study, as the dominant and easily accessible muscles of the arm for surface electromyography measurements, we process the EMG activity of the Biceps Brachii (BB) $\left(P_{B}\right)$ and Triceps Brachii (TB) $\left(P_{T}\right)$ muscles to calculate $a_{c c}(p)=c_{1}+c 2\left(P_{B}+P_{T}\right)$, with $c_{1}$ and $c_{2}$ being constant coefficients referring to the intrinsic muscle stiffness component. By rearranging the above equations we obtain

$$
K_{c}(p, q)=J^{+T}(q)\left[J_{m}^{T}(q) a_{c c}(p) K_{s} J_{m}(q)\right] J^{+}(q) .
$$

The parameters of such models are subject-specific and must be identified off-line. To achieve this, as described in [19], the endpoint stiffness $\left(K_{c}(p, q)\right)$ of the human arm was estimated in various arm pose $(q)$ and activation levels of the arm muscles $(P)$. The stiffness matrix was estimated by applying stochastic position perturbations and measurements of the restoring forces. The estimated stiffness matrices and the measured data (muscle activities $(P)$ and arm and muscle Jacobians calculated from $q$ ) can be consequently used for the identification of the model parameters by minimizing the norm

$$
\left\|J_{m}^{T}(q) a_{c c}(p) K_{s} J_{m}(q)-J^{T}(q) K_{c}(p, q) J(q)\right\|,
$$

with $J_{m} \in \mathbb{R}^{8 \times 7}, K_{s} \in \mathbb{R}^{8 \times 8}, J \in \mathbb{R}^{3 \times 7}$, and $K_{c} \in \mathbb{R}^{3 \times 3}$ (only translational components of the stiffness matrix). In this model, we exploit arm muscle length functions of eight dominant muscles acting on the shoulder, elbow and wrist joint to model the moment arms of the muscles as functions of the corresponding joint angles (see [25]). Selected eight muscles are the anterior (DELT1) and posterior (DELT3) portions of Deltoid, Brachialis (BRA), Brachioradialis (BRD), the long (BIClong) and short (BICshort) portions of Biceps, and the long (TRIlong) and lateral (TRIlat) portions of Tricpes, which present dominant effects in generation of the torque profiles in the arm joints. Therefore, 10 unknown parameters $\left(K_{s}, c_{1}\right.$, and $c_{2}$ ) must be identified, which define the minimum number of required trials for the calibration experiments. Based on this number, the total number of trials was divided into the calibration and test trials for the validation of the identified model. The identified matrix $K_{s}$ represents the coordinated contributions of the selected muscles to the endpoint stiffness variations. The other two components $c_{1}$, and $c_{2}$ are used for the definition of the scalar value $a_{c c}$, that represents the active contribution of muscular activities to the modifications of the volume of the endpoint stiffness matrix [19].

Once the model parameters are identified, (2) can be utilized for the real-time computation of the arm endpoint stiffness profile using electromyography signals of one antagonistic pair of muscles and the tracking of the arm triangle (please refer to [19] for details). The EMG signals are acquired by the wireless Delsys Trigno system (Delsys Inc.), at $1 \mathrm{kHz}$. The processing (filtering and normalization to maximum voluntary contraction (MVC)) is performed on-line. The tracking of the arm triangle is achieved by 11 Flex-3 cameras of the Optitrack system (NaturalPoint, Inc.) by attaching three rigid-body markers to the shoulder, elbow and wrist of the human hand, at 100 $\mathrm{Hz}$. Our real-time model enables the master to modify the direction of the endpoint stiffness ellipsoid by changing the arm posture in an intuitive manner, while being capable of adjusting its volume by increasing the co-contraction of the dominant arm muscles. As a result, teleoperated tasks which require significant modulation of the endpoint stiffness and force can be executed effectively and naturally.

\section{A Wearable Approach to Dynamic Remote CONTROL OF A SYNERGY DRIVEN ROBOTIC HAND}

\section{A. A Wearable Hand Pose Reconstruction Ssystem: Optimal Design and Under-sensing}

The position of the Pisa/IIT SoftHand was controlled based on the user's hand posture acquired through a wearable sensing glove. This glove [18] was endowed with five texile goniometers which can measure five joint angles, see Fig. 3. This glove has also been integrated with a tactile sensing glove to provide 
a tool to retrieve both kinematic and force information of human hand in grasping tasks [26]. Textile goniometers consist

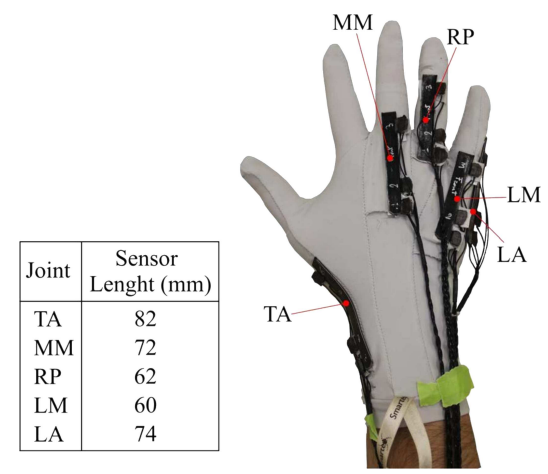

Figure 3: Glove with knitted piezoresistive fabrics (KPF) sensors on the joints of interest.

of two piezoresistive layers connected through an electricallyinsulating layer. The sensing layers of the textile goniometers were fabricated using knitted piezoresistive fabrics (KPFs). The output of the sensors is the electrical resistance difference computed from the two sensitive layers and is proportional to the flexion angle [27], see Fig. 4. The distribution of the

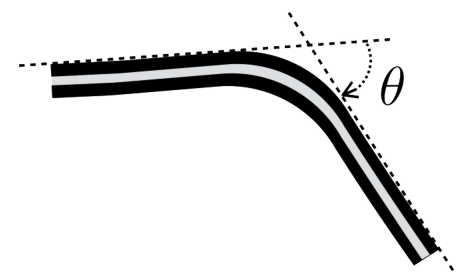

Figure 4: Representation of a double-layer textile goniometer. The two external stripes represent the piezoresistive layers. The grey line is the electrically-insulating layer. The bending angle $(\theta)$, represented by the angle between the tangent planes to the goniometer extremities (black dashed stripe), is proportional to the difference of the resistance $(\Delta R)$ of the sensing layers.

sensors on the glove was optimal in a Bayesian sense such that it maximizes the information on the actual hand posture.

The theoretical work that led to the definition of the design guidelines was laid out in [28]: the idea was to exploit hand joint covariation schemes in grasping tasks, or hand synergies [13] as a priori information to complete hand pose from a limited number of (noisy) measures and, at the same time, to decide how and whereto place sensors on the glove taking into account the knowledge on how humans most frequently use their hands in grasping tasks.

More specifically, five KPF goniometers were specifically built and integrated into the glove to fulfill the design requirements (i.e., sensor length) and to measure the following joint angles, according to the kinematic model in [13], i.e., TA, MM, RP, LA and LM (Thumb Abduction, Middle Metacarpal, Ring Proximal, Little Abduction, Little Metacarpal, respectively). Such a sensor placement was inspired by the optimal design guidelines in [28]: the measurements KPFs provide were then completed through synergy-based estimation techniques [29] to obtain hand posture reconstruction according to a 19DOF model. More details on the sensing glove can be found in [18].

\section{B. Kinematic Mapping: from Human Hand to Pisa/IIT Soft- Hand}

Once the hand pose of the user is reconstructed, it is used to control the position of the Pisa/IIT SoftHand, which is built to move without obstacles along the vector of the first human postural synergy $S$ (in our case $S \in \mathbb{R}^{19}$ ) - which also represents the reference configuration towards which the real hand position is attracted and, at the same time repelled from, due to the interaction and grasping with the external object, according to the soft synergy model [30].

Since the SoftHand is commanded to move along the first human postural synergy, for this experiment, we need to estimate the value of $\sigma$, i.e. the synergy intensity [30], which should be commanded to the SoftHand motor.

Vector $S$ is implemented in the SoftHand through a careful design of spring elements and pulley trains, resulting in a coordinated closure of all joint angles. To get value $\sigma$

$$
\sigma=S^{T} x
$$

where vector $x$ contains the reconstructed human hand joints.

This value needs to be correlated with $\mathrm{SH}$ motor position: to do it, we first get $\sigma_{\max }$ corresponding to the maximum closure of human hand and related it to motor position (in ticks). The other intermediate $\sigma$ values can be obtained via simple proportion. This reconstruction provides an effective way to command synergy-based robotic hands and can be extended to systems with more degrees of actuation, see e.g. [31].

\section{Teleimpedance for Intuitive Control of the Robotic Hand}

Following the implementation of teleimpedance control for teleoperation of a robotic arm described above, we explored the translation of this approach to the control of a synergy driven robotic hand, the Pisa/IIT SoftHand ([17]). The reference configuration commanded to the hand is the difference of the two EMG signals from FDS and EDC, as previously mentioned. These signals were acquired through two surface EMG electrodes by Trigno System. To modulate the stiffness of the hand, we used an EMG driven P-gain modulation. In other terms, the proportional gain of the PID controller of the position control of the $\mathrm{SH}$ motor increased with increasing amplitude of the EMG signal with the smallest amplitude, normalized to the EMG signal highest amplitude. Upper and lower bounds for the proportional gain were estimated through pilot data by selecting the range of values in which the SH moved smoothly until reaching the reference position. Accordingly, SH movement velocity and impedance were modified by changing the proportional gain value. More details can be found in [32]. 


\section{Wearable Haptic Device for Force Feedback}

To convey information about the grasping force produced by the Pisa/IIT SoftHand we used the Clenching Upper-limb Force Feedback wearable device (hereinafter referred to as CUFF) for distributed mechano-tactile stimulation of normal and tangential skin forces. The device is described with more details in [16].

CUFF device can be worn by the users on their forearm and is endowed with two independently controlled DC motors. The basic design of the device is based on an elastic belt wrapped around the user's limb. For this study, the motors spin in opposite directions to tighten or loosen the band on the arm in accordance with estimated grasp force.

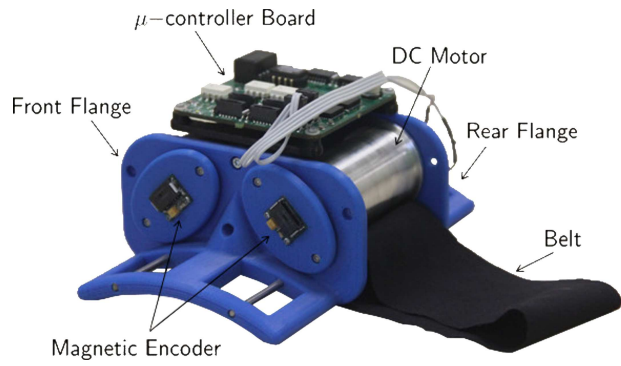

(a)
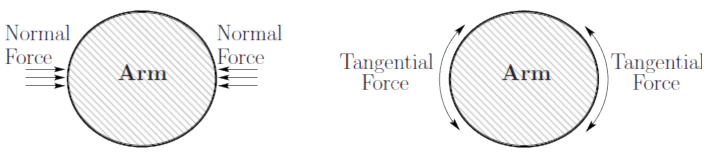

(b)

Figure 5: Device overview and working modes.

In Fig. 5 the real prototype and a 3D render of the CUFF system are shown, highlighting some details of its mechanical implementation. The CUFF weights $\approx 230 \mathrm{~g}$, while its overall dimensions are $12.4 \times 7.0 \times 5.8 \mathrm{~cm}$.

One possible application of CUFF is to enhance haptic interaction with the environment, in particular as a haptic feedback device for robotic/prosthetic hands, with the goal of achieving better grasp stability by conveying grip force information [17]. The Pisa/IIT SoftHand was equipped with a custom-made $\mu$-controller, which controls the opening/closing level of the hand in position by acting on the current that drives the motor. The complete scheme of the control is represented in Fig. 2. The basic idea is to use the hand motor current to have a rough estimate of the applied force to the external environment. This approach is motivated by the fact that there is a net difference in the motor current, in free motion (maximum value of $\approx 800 \mathrm{~mA}$ ) or when the robotic hand grasps an external object (maximum value of $\approx 1200$ $\mathrm{mA})$. The CUFF is then controlled through the residual current $r_{I}$, defined as the difference between the current absorbed by the SoftHand motor and the current reconstructed. The reconstructed current represents the motor current in free hand motion, which will be subtracted from the current sensed by the $\mu$-controller. More details can be found in [16].

\section{INTEGRATION AND EXPERIMENTAL TASK}

In our experimental setup, we integrated the aforementioned techniques, i.e. teleimpedance, sensing glove and forcefeedback device. We implemented two control loops: one controlled the sensing glove, the Pisa/IIT SoftHand (both in position and stiffness) and managed the force feedback. The other one controlled the Kuka robot, through a developed Cartesian impedance controller to replicate the masters' arm endpoint trajectories and stiffness. EMG signals were acquired by a third computer that sent these values in broadcast on a local wired network. The two control loops, running on two distinct computers, took data of interest directly from the network. These two loops run at a frequency of $100 \mathrm{~Hz}$ and $200 \mathrm{~Hz}$, respectively. During the experiments, we asked participants to control the pose and stiffness of the Pisa/IIT SoftHand mounted on Kuka. The arm teleimpedance control (described in section 2) was used to command the position of the robotic arm and to regulate the impedance of the system online. The task consisted in grasping a drilling tool and using it to drill a block of autoclaved aerated concrete. The task was considered successful if participant was able to grasp the tool placed on a table, use it to drill the block (to produce a hole with length of at least $4 \mathrm{~cm}$ ) and remove the drill from the hole.

Ten right-handed volunteers ( 8 males and 2 females, age $27.4 \pm 2.63)$ took part in the experimental tests. All participants in these studies gave informed consent to perform the experiments. No subjects reported physical limitations that would affect their ability to perform the task. Average arm length is $29.47 \mathrm{~cm}$ with a standard deviation of $2.41 \mathrm{~cm}$ from the shoulder to the elbow, and $24.02 \mathrm{~cm}$ with standard deviation $4.06 \mathrm{~cm}$ from the elbow to the wrist. Average hand measures are $10.21 \pm 0.93 \mathrm{~cm}$ width (from the tip of the thumb to the tip of the little finger in flat hand posture) and $18.48 \pm 0.79 \mathrm{~cm}$ length (from the wrist to the middle finger tip). For any subject a fast calibration for the sensing glove (see section 3) and for the teleimpedance controllers for the arm and the hand was performed (see sections 2 and 3, respectively).

Six experimental conditions were considered: (i) Low Stiffness (LS), (ii) High Stiffness (HF), (iii) teleimpedance (TI), with and without the usage of the cuff force feedback, (C) and (NC), respectively. In the LS condition the stiffness of the $\mathrm{SH}$ was also low and we set the P-gain of the $\mathrm{SH}$ controller as 0.005 , and the Cartesian stiffness of the KUKA robot was set to $800 \frac{\mathrm{N}}{\mathrm{m}}$ and $50 \frac{\mathrm{Nm}}{\mathrm{rad}}$ in all translational and rotational directions, respectively. For the HS condition the P-gain of the SH was set to 0.05, with the KUKA Cartesian stiffness values of $2000 \frac{\mathrm{N}}{\mathrm{m}}$ and $200 \frac{\mathrm{Nm}}{\mathrm{rad}}$ in all translational and rotational directions, respectively. These represent lower and upper bound guaranteeing a stable performance. In the TI condition the P-gain and the robot endpoint stiffness profile were modulated as described in Section before.

At the end of the experiments, we asked subjects to answer the following questions according to a seven-point bipolar Likert-like scale:

1) I had the feeling of performing better while receiving force feedback by the cutaneous device 
2) I had the feeling of performing better while modulating impedance of end effector

3) I was feeling uncomfortable while using teleimpedance with cutaneous device

4) Teleimpedance control was intuitive

5) I felt hampered by the cutaneous device

6) It was easy to move my hand and fingers while wearing the sensing device

7) Please rate your impression on how close the robot behaved as an extension of your body (including the feeling to directly interact with the remote environment to assess somehow transparency)

where score 1 means completely disagree and 7 completely agree.

\section{RESULTS}

As performance evaluation metrics, we considered: success in task accomplishment and modulation of EMG signals and interaction forces. We will discuss the main experimental outcomes in this section, considering the effect of teleimpedance modulation and force feedback. A video of an exemplary trial performed with teleimpedance control and force feedback can be found in the accompanying video.

\section{A. Success Rate}

In Fig. 6 we report the success rate for the different conditions. Regarding conditions TI, LS, HS we can observe that TI exhibits the highest success percentage (83.3\%), although high stiffness also provides high success rate $(78.3 \%$ w.r.t. $60.0 \%$ for LS).

We have performed $\chi^{2}$ non-parametric statistical tests considering the relative frequency of succeeding trials between the three different experimental conditions (Teleimpedance, Low stiffness, High stiffness) and for the two factors (CUFF and No CUFF).

Considering Teleimpedence condition, the contingency table of observed frequencies is associated with a $p<0.085\left(\chi^{2}=\right.$ $3, d f=1)$ as calculated considering the Yates correction for the $\chi^{2}$ calculation due to the low value of expected frequencies, meaning that in this experimental condition the use of CUFF device tends to increase the chances of success trial rate. Nevertheless, we can only claim on this "trend" because of the formal acceptance of the null-hypothesis considering a statistical significance of 5\%. Likely, a higher number of subjects involved in our experimental protocol would allow reaching a proper statistical significance.

Considering low-stiffness condition, the contingency table of observed frequencies is associated with a $p<0.05$ $\left(\chi^{2}=4.44, d f=1\right)$, meaning that in this experimental condition the use of CUFF device significantly increases the chances of success trial rate. This could be ascribed to the lower force produced by the SoftHand in this case compared to the other two ones. In this condition, the information conveyed by the CUFF is essential to make the subject aware that the control he/she is acting is not sufficient for lifting, carrying and handling the driller and hence can increase the grasping force for a successful task completion.
Considering high-stiffness condition, the contingency table of observed frequencies is associated with a $p>0.05\left(\chi^{2}=\right.$ $0.884, d f=1)$, meaning that in this experimental condition the use of CUFF device does not increase the chances of success trial rate. This may be due to the fact that in High stiffness condition the proportional gain of the SH control is very high, resulting in fast and strong movements of the hand itself. These movements generate high grasping forces that can be difficulty regulated even with the usage of the CUFF.

Importantly, regardless of the experimental condition, the contingency table of observed frequencies is associated with a $p<0.005\left(\chi^{2}=8.32, d f=1\right)$, demonstrating that in a generic scenario the use of the CUFF device significantly increases the chances of success trial rate.

We then investigated differences in the trial success rate regardless of the use of the CUFF device. In this case, the contingency table of observed frequencies is associated with a $p<0.01\left(\chi^{2}=9.39, d f=2\right)$, meaning that the specific experimental condition does influence the trial success rate. We then performed $\chi^{2}$ post-hoc test considering Bonferroni correction of the statistical significance, observing that the teleimpedence significantly increases the change of trial success rate with respect to the low-stiffness condition $\left(p<0.005, \chi^{2}=8.04\right.$, $d f=1$ ). Other multiple comparisons do not reach the formal statistical significance (as associated with corrected $p>0.05$ ), although it is worthwhile noting that the low-stiffness vs. highstiffness comparison is associated with a corrected $p<0.06$ $\left(\chi^{2}=4.73, d f=1\right)$, meaning that the high-stiffness condition tends to increase the change of trial success rate with respect to the low-stiffness condition. The non-statistical difference between Teleimpedance and the High stiffness condition could be explained by the fact that for this particular task, the High stiffness condition generates very precise movements that led to a successful trial execution. However, the integrated usage of teleimpedance and Cuff produces a different modulation of the interaction forces, which are generally lower w.r.t the High Stiffness case and suitably adapted to the different phases of the trial. This would result in safer interactions and likely to a reduced fatigue in subjects for longer tasks (see Section VII-E)

\begin{tabular}{|c|c|c|c|c|c|c|}
\hline \multicolumn{7}{|c|}{ Percentages succeeded trial } \\
\hline \multirow[b]{2}{*}{ Subj } & \multicolumn{2}{|c|}{ teleimpedance } & \multicolumn{2}{|c|}{ Low stiffness } & \multicolumn{2}{|c|}{ High stiffness } \\
\hline & CUFF & No CUFF & CUFF & No CUFF & CUFF & No CUFF \\
\hline 1 & $100,0 \%$ & $100,0 \%$ & $100,0 \%$ & $100,0 \%$ & $66,7 \%$ & $100,0 \%$ \\
\hline 2 & $100,0 \%$ & $66,7 \%$ & $100,0 \%$ & $66,7 \%$ & $100,0 \%$ & $100,0 \%$ \\
\hline 3 & $66,7 \%$ & $33,3 \%$ & $66,7 \%$ & $0,0 \%$ & $66,7 \%$ & $100,0 \%$ \\
\hline 4 & $100,0 \%$ & $100,0 \%$ & $33,3 \%$ & $0,0 \%$ & $33,3 \%$ & $66,7 \%$ \\
\hline 5 & $100,0 \%$ & $66,7 \%$ & $33,3 \%$ & $0,0 \%$ & $100,0 \%$ & $66,7 \%$ \\
\hline 6 & $100,0 \%$ & $66,7 \%$ & $100,0 \%$ & $33,3 \%$ & $100,0 \%$ & $0,0 \%$ \\
\hline 7 & $100,0 \%$ & $100,0 \%$ & $100,0 \%$ & $100,0 \%$ & $100,0 \%$ & $66,7 \%$ \\
\hline 8 & $66,7 \%$ & $0,0 \%$ & $33,3 \%$ & $0,0 \%$ & $66,7 \%$ & $66,7 \%$ \\
\hline 9 & $100,0 \%$ & $100,0 \%$ & $66,7 \%$ & $66,7 \%$ & $100,0 \%$ & $66,7 \%$ \\
\hline 10 & $100,0 \%$ & $100,0 \%$ & $100,0 \%$ & $100,0 \%$ & $100,0 \%$ & $100,0 \%$ \\
\hline Tot & $93,3 \%$ & $73,3 \%$ & $73,3 \%$ & $46,7 \%$ & $83,3 \%$ & $73,3 \%$ \\
\hline Tot group & 83 & & 60 & $0 \%$ & & \\
\hline
\end{tabular}

Figure 6: Success percentage of task accomplishment for the different conditions. 


\section{B. Subjective Evaluation}

The scores provided by 10 participants (reported in Table I) were analyzed through descriptive and inferential statistics.

Analyzing the data, we can conclude that cutaneous device and teleimpedance increase subjective evaluation of task performance $(\mathrm{Q} 1, \mathrm{Q} 2)$. In other terms, participants had the impression to perform better when teleimpedance control and force feedback were provided. Furthermore, the integration of teleimpedance and cuff device was perceived as comfortable by the subjects, who did not feel hampered by the cutaneous device (Q3, Q5). The sensing glove for HPR was evaluated as highly wearable, imposing minimal constraints to fingers and hand movements (Q6). Regarding the transparency of the system to the user (Q7), the usersrated the perception of the robot as an extension of their body when Teleimpedance and CUFF were used together providing a very high score.

\section{EMG modulation and Hand Interaction Forces}

This section reports on the effect of feedback (CUFF) device on the modulation of EMG signals of the EDC and FDS muscles, and the grasping forces, as a consequence. Figs. 7 and 8 illustrate typical results of the EMG activities and the corresponding grasping interaction forces of the SoftHand under teleimpedance control without and with the haptic feedback, respectively. As observed, haptic interface enables the user to effectively modulate the EMG activities and the grasp forces to achieve a stable grasp in different phases of the task.
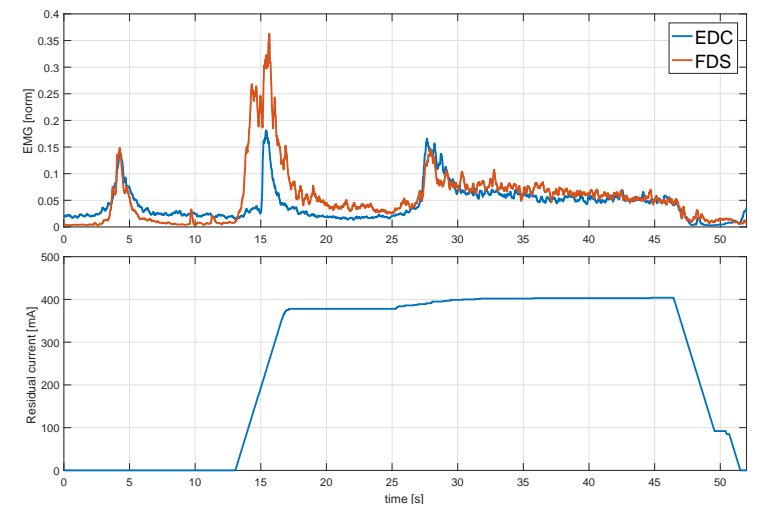

Figure 7: Modulation of EDC and FDS signals and SoftHand grasping force (expressed in terms of residual current) when the user (Subject 1) did not use CUFF device.

\section{EMG modulation and Endpoint Stiffness (changing the P- gain)}

This section illustrates the results of the regulation of the robot endpoint stiffness through control of CMS (contribution of muscular activations) and CDS (through arm geometry).The impact of varying the hand stiffness (by changing the $\mathrm{P}$ gain) and the Cartesian stiffness profiles of KUKA can be assessed on the dynamic performance of the system. Indeed, we do not expect to see any performance degradation using various $\mathrm{P}$ gains or Cartesian stiffness profiles in terms of trajectory

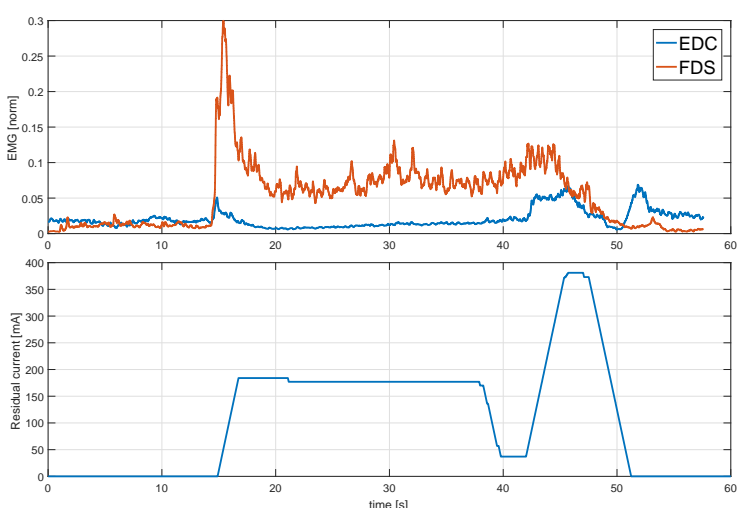

Figure 8: Modulation of EDC and FDS signals and SoftHand grasping force (expressed in terms of residual current) when the user (Subject 1) wore and used CUFF device.

tracking in free space (assuming a reasonable compensation of system uncertainties such as friction). Nevertheless, the force response of the two systems (hand and robot arm) to the environmental displacements would clearly differ, and determined by the choice of the parameters.

The objective here is to illustrate that certain endpoint impedance profiles and hence certain gains (for hand and KUKA) provide the best performance for certain physical interaction scenarios, as is the case of a drilling task. Hence, since the best performance relies on the choice of gains, we use a human-in-the-loop system to tune them online, intuitively. Typical results of the teleimpedance control with haptic feedback are illustrated in Figs. 9(a) and 9(b) for two typical subjects, respectively. As illustrated in the plots, the operators could modulate the volume of the Cartesian stiffness profile (a coordinated increase in the matrix components) by applying co-contractions, in different phases of the task.

In addition, in robot arm control, if the control of the endpoint stiffness geometry was necessary, the proposed method enables the operator to use the effect of configuration to modulate the stiffness in certain axes of the Cartesian stiffness (e.g. while drilling the arm is extended on $x$ direction to achieve a stiffer profile in $x$ even in small muscular activation levels) intuitively. Hence, potential misalignments in other directions would not achieve unnecessary high interaction forces. This explains the change of endpoint stiffness in similar activation levels of the muscles but different arm poses of the operator. The operators ability to modulate the endpoint stiffness of the KUKA robot (and the restoring forces as a consequence) according to the task needs, led to an increase in the success rate.

\section{E. Interaction Forces}

Figure 10 illustrates typical acquired interaction forces at the KUKA end-effector for the three control modes (LS, HS, TI) during drilling task. As observed in the plots, while interaction forces are low in the Low Stiffness case, the time of the execution is very long compared to the rest of the modes. Stiff case, on the other hand, achieved unnecessary high interaction force in directions other than drilling (e.g. in $y$ direction) 


\begin{tabular}{|c|c|c|c|c|}
\hline & Questions & Median & I.Q.R. & CI m. $95 \%$ \\
\hline Q1 & I had the feeling of performing better while receiving force feedback by the cutaneous device. & 6 & 1 & $(5,6)$ \\
\hline Q2 & I had the feeling of performing better while modulating impedance of end effector. & 6 & 1 & $(5,7)$ \\
\hline Q3 & I was feeling uncomfortable while using teleimpedance with cutaneous device. & 2 & 0 & $(1,3)$ \\
\hline Q4 & Teleimpedance control was intuitive. & 6 & 0 & $(5,6)$ \\
\hline Q5 & I felt hampered by the cutaneous device. & 2 & 0 & $(1,2)$ \\
\hline Q6 & It was easy to move my hand and fingers while wearing the sensing device. & 6 & 0 & $(4,7)$ \\
\hline Q7 & Please rate your impression on how close the robot behaved as an extension of your body. & 6 & 1 & $(5,7)$ \\
\hline
\end{tabular}

Table I: These statements, presented in random order, were rated by the subjects using a 7-point Likert scale (1: Strongly disagree, 7: Strongly agree). Data from the statistical analysis are showed. Q7 is referred to the Teleimpedance condition with CUFF feedback.

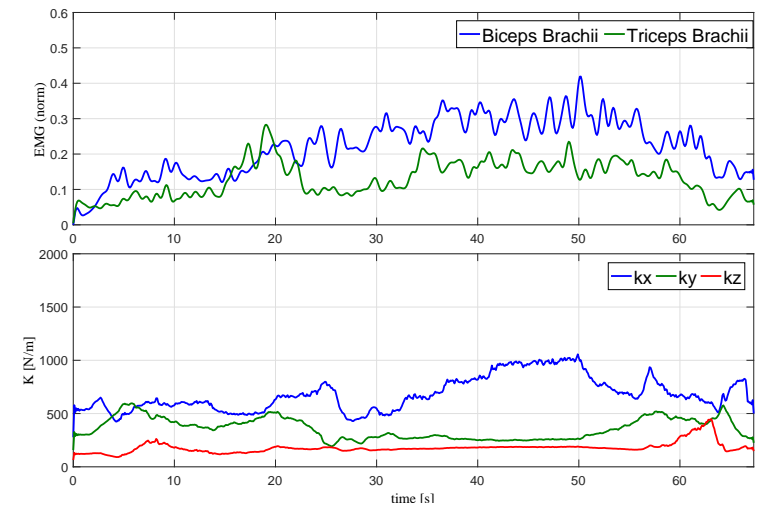

(a)

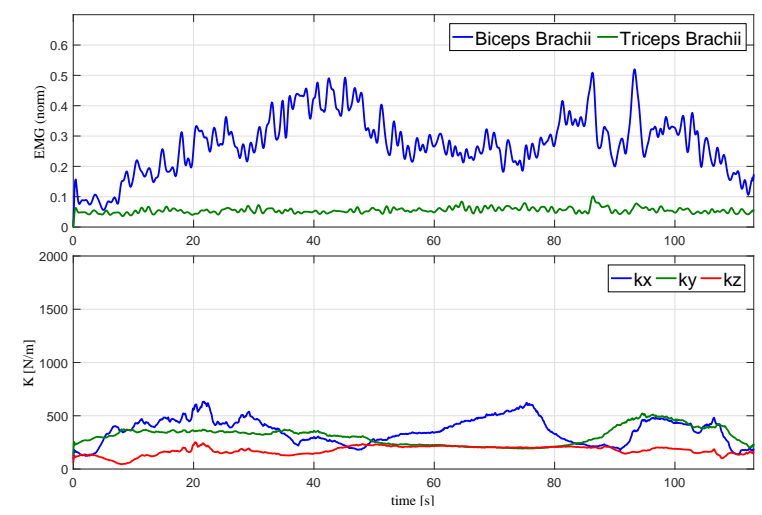

(b)

Figure 9: Processed (filtered and normalized) electromyography signals of the Biceps Brachii and Triceps Brachii muscles are illustrated in the upper plot in the two subfigures during the teleimpedance experiment. The resulting human arm endpoint stiffness (that is achieved in KUKA endpoint using the Cartesian impedance controller of the robot), in KUKA frame of reference is illustrated in the lower plot of the two subfigures for the subject 1 (a) and subject 4 (b) for the Teleimpedance controller with haptic feedback. which could cause damage for the tool or the environment. On the other case, Teleimpedance control mode enables to regulate interaction forces according to the task phase and its requirement, as depicted in the figure.

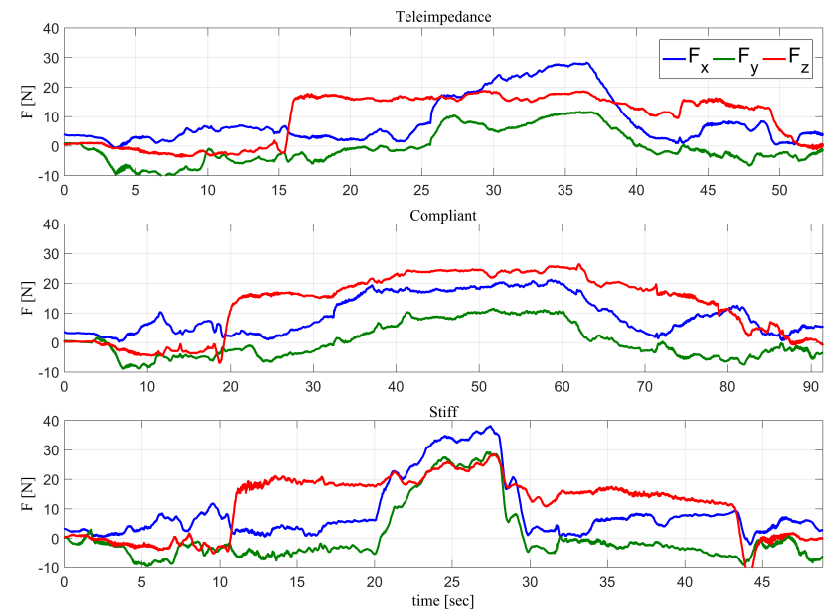

Figure 10: Typical acquired interaction forces at the KUKA end-effector for the three control modes. All three modes include haptic feedback.

\section{CONClusions AND Future WORKS}

In this paper, we discuss the results of a human-robot teleoperation in a cooperative object manipulation task, leveraging upon wearablity paradigm and the generalized simplification approach of human synergies. The task is a drilling task, where a human user was required to tele-operate a robotic synergy-inspired hand-arm system, endowed with the Pisa/IIT SoftHand, in order to grasp and use a driller. The correct task accomplishment requires advanced action-perception skills, which include both knowledge about the object properties and the execution of motor primitives, but without increasing the complexity for sensing and control on the robotic side.

To perform this action, we developed a system that integrates in a coherent manner under-sensing and wearable haptic feedback devices and augmented tele-operation methods based on teleimpedance, which enables to simply, safely and stably control the position and the stiffness of a robotic hand and arm system in the synergy space. Results from a preliminary experimental validation carried out with human participants 
show that the usage of teleimpedance and force feedback seems to increase the success rate and was perceived by participants as a highly valuable and intuitive aid for task accomplishment. Teleimpedance mode with haptic feedback enabled subjects to effectively regulate EMG signals and the interaction forces of the Pisa/IIT SoftHand. Furthermore, the wearability of the sensing glove used to control the position of the robotic hand along the first human grasping synergy was perceived by participants as significantly high. Conclusions that can be drawn are that the presented system seems to increase effectiveness, intuitiveness and comfort during collaborative tasks performed by a human user and a robot, ensuring stability and naturalness of HRI for synergy-inspired artificial devices. It is also important to observe that these techniques can be successfully applied and extended to robotic devices with additional degrees of actuation, enabling a simplified and effective human-robot communication.

Future works will focus on further testing this system with a greater number of participants and evaluating other modalities for HPR, e.g., visual based, as well as the effect of other feedback modes, e.g., vibrotactile. Wearability of the overall system will be also pushed further. Envisioned applications can be in medical robotics, entertainment and amusement culture and tele-operation in hazardous and remote environments.

\section{ACKNOWLEDGMENTS}

The authors would like to thank Marta Lorenzini and Pietro Balatti for their help in performing the experiments.

\section{REFERENCES}

[1] D. A. Lawrence, "Stability and transparency in bilateral teleoperation," IEEE transactions on robotics and automation, vol. 9, no. 5, pp. 624$637,1993$.

[2] B. Hannaford, "A design framework for teleoperators with kinesthetic feedback," IEEE transactions on Robotics and Automation, vol. 5, no. 4, pp. 426-434, 1989.

[3] J.-H. Ryu, D.-S. Kwon, and B. Hannaford, "Stability guaranteed control: Time domain passivity approach," IEEE Transactions on Control Systems Technology, vol. 12, no. 6, pp. 860-868, 2004.

[4] L. Huijun and S. Aiguo, "Virtual-environment modeling and correction for force-reflecting teleoperation with time delay," IEEE Transactions on Industrial Electronics, vol. 54, no. 2, pp. 1227-1233, 2007.

[5] H. Qin, A. Song, Y. Liu, G. Jiang, and B. Zhou, "Design and calibration of a new 6 dof haptic device," Sensors, vol. 15, no. 12, pp. 31293 $31313,2015$.

[6] R. E. Schoonmaker and C. G. L. Cao, "Vibrotactile force feedback system for minimally invasive surgical procedures," in 2006 IEEE International Conference on Systems, Man and Cybernetics, vol. 3, Oct 2006, pp. 2464-2469.

[7] D. Prattichizzo, C. Pacchierotti, and G. Rosati, "Cutaneous force feedback as a sensory subtraction technique in haptics," IEEE Transactions on Haptics, vol. 5, no. 4, pp. 289-300, 2012.

[8] X. Xu, A. Song, D. Ni, H. Li, P. Xiong, and C. Zhu, "Visual-haptic aid teleoperation based on 3-d environment modeling and updating," IEEE Transactions on Industrial Electronics, vol. 63, no. 10, pp. 6419-6428, 2016.

[9] A. Ajoudani, Transferring Human Impedance Regulation Skills to Robots. Springer, 2016.

[10] C. Della Santina, M. Bianchi, G. Grioli, M. G. Catalano, M. Garabini, and A. Bicchi, "Feedback and feedforward control for soft robots," Robotics and Automation Magazine, IEEE, In Press.

[11] M. Bianchi, "A fabric-based approach for wearable haptics," Electronics, vol. 5 , no. 3, p. 44, 2016

[12] C. Pacchierotti, F. Chinello, M. Malvezzi, L. Meli, and D. Prattichizzo, "Two finger grasping simulation with cutaneous and kinesthetic force feedback," in International Conference on Human Haptic Sensing and Touch Enabled Computer Applications. Springer, 2012, pp. 373-382.
[13] M. Santello, M. Flanders, and J. F. Soechting, "Postural hand synergies for tool use," The Journal of Neuroscience, vol. 18, no. 23, pp. $10105-$ $10115,1998$.

[14] M. Santello, M. Bianchi, M. Gabiccini, E. Ricciardi, G. Salvietti, D. Prattichizzo, M. Ernst, A. Moscatelli, H. Jorntell, A. Kappers, K. Kyriakopulos, A.Albu-Schaeffer, C. Castellini, and A. Bicchi, "Hand synergies: Integration of robotics and neuroscience for understanding the control of biological and artificial hands," Physics of Life Reviews, vol. 17, pp. 1-23, 07/2016 2016. [Online]. Available: http://www.sciencedirect.com/science/article/pii/S1571064516000269

[15] M. G. Catalano, G. Grioli, E. Farnioli, A. Serio, C. Piazza, and A. Bicchi, "Adaptive synergies for the design and control of the pisa/iit softhand," The International Journal of Robotics Research, vol. 33, no. 5, pp. 768782, 2014

[16] S. Casini, M. Morvidoni, M. Bianchi, M. Catalano, G. Grioli, and A. Bicchi, "Design and realization of the cuff - clenching upperlimb force feedback wearable device for distributed mechano-tactile stimulation of normal and tangential skin forces," in Intelligent Robots and Systems (IROS), 2015 IEEE/RSJ International Conference on, Sept 2015, pp. $1186-1193$.

[17] A. Ajoudani, S. B. Godfrey, M. Bianchi, M. G. Catalano, G. Grioli, N. Tsagarakis, and A. Bicchi, "Exploring teleimpedance and tactile feedback for intuitive control of the pisa/iit softhand," IEEE transactions on haptics, vol. 7, no. 2, pp. 203-215, 2014.

[18] S. Ciotti, E. Battaglia, N. Carbonaro, A. Bicchi, A. Tognetti, and M. Bianchi, "A synergy-based optimally designed sensing glove for functional grasp recognition," Sensors, vol. 16, no. 6, p. 811, 2016.

[19] A. Ajoudani, C. Fang, N. G. Tsagarakis, and A. Bicchi, "A reducedcomplexity description of arm endpoint stiffness with applications to teleimpedance control," in Intelligent Robots and Systems (IROS), 2015 IEEE/RSJ International Conference on. IEEE, 2015, pp. 1017-1023.

[20] T. Milner, "Contribution of geometry and joint stiffness to mechanical stability of the human ijm," Experimental Brain Research, vol. 143, pp. 515-519, 2002.

[21] S. L. Delp, F. C. Anderson, A. S. Arnold, P. Loan, A. Habib, C. T John, E. Guendelman, and D. G. Thelen, "Opensim: open-source software to create and analyze dynamic simulations of movement," IEEE transactions on biomedical engineering, vol. 54, no. 11, pp. 1940-1950, 2007.

[22] E. Van Zuylen, C. Gielen, and J. D. Van Der Gon, "Coordination and inhomogeneous activation of human arm muscles during isometric torques," Journal of Neurophysiology, vol. 60, no. 5, pp. 1523-1548, 1988.

[23] R. Osu and H. Gomi, "Multijoint muscle regulation mechanisms examined by measured human arm stiffness and emg signals," Journal of neurophysiology, vol. 81, no. 4, pp. 1458-1468, 1999.

[24] M. T. Turvey, "Action and perception at the level of synergies," Human movement science, vol. 26, no. 4, pp. 657-697, 2007.

[25] P. Pigeon, L. Yahia, and A. G. Feldman, "Moment arms and lengths of human upper limb muscles as functions of joint angles," Journal of biomechanics, vol. 29, no. 10, pp. 1365-1370, 1996.

[26] M. Bianchi, R. Haschke, G. Büscher, S. Ciotti, N. Carbonaro, and A. Tognetti, "A multi-modal sensing glove for human manual-interaction studies," Electronics, vol. 5, no. 3, p. 42, 2016.

[27] A. Tognetti, F. Lorussi, G. Dalle Mura, N. Carbonaro, M. Pacelli, R. Paradiso, and D. De Rossi, "New generation of wearable goniometers for motion capture systems," Journal of neuroengineering and rehabilitation, vol. 11, no. 1, p. 1, 2014.

[28] M. Bianchi, P. Salaris, and A. Bicchi, "Synergy-based hand pose sensing: Optimal glove design," The International Journal of Robotics Research, vol. 32, no. 4, pp. 407-424, 2013.

[29] - "Synergy-based hand pose sensing: Reconstruction enhancement," The International Journal of Robotics Research, vol. 32, no. 4, pp. 396406, 2013.

[30] A. Bicchi, M. Gabiccini, and M. Santello, "Modelling natural and artificial hands with synergies," Philosophical Transactions of the Royal Society of London B: Biological Sciences, vol. 366, no. 1581, pp. 31533161, 2011.

[31] C. Della Santina, G. Grioli, M. Catalano, A. Brando, and A. Bicchi, "Dexterity augmentation on a synergistic hand: The pisa/iit softhand+," in Humanoid Robots (Humanoids), 2015 IEEE-RAS 15th International Conference on. IEEE, 2015, pp. 497-503.

[32] S. Fani, M. Bianchi, S. Jain, J. S. P. Neto, S. Boege, G. Grioli, A. Bicchi, and M. Santello, "Assessment of myoelectric controller performance and kinematic behavior of a novel soft synergy-inspired robotic hand for prosthetic applications," Frontiers in Neurorobotics, vol. 10, 2016. 